\title{
A Survey on Image Restoration by Comparing the Different Deblurring Process and Filtration
}

\author{
Navpreet Kaur ${ }^{1}$, Varun Sanduja ${ }^{2}$ \\ ${ }^{1}$ Student, Electronics and Communication Department, CGCTC, Jhanjeri, Punjab, India \\ ${ }^{2}$ Assistant Professor, Electronics and Communication Department, CGCTC, Jhanjeri, Punjab, India
}

\begin{abstract}
Image processing is an important component of modern technologies as it provides the improvement in pictorial information for human interpretation and processing of image data for storage, transmission and representation autonomous machine perception. This paper focused on image restoration which is sometimes referred to image deblurring and filtering. Image restoration is concerned with the reconstruction of blur parameters of the uncorrupted image from a blurred and noisy one. Image deblurring refers to procedures that attempt to reduce the blur amount in a blurry image and grant the degraded image an overall sharpened appearance to obtain a clearer image. In this paper, the various kind of noise are added and then deblurring process is used to obtain a blurred image. After this image filtering is also implemented for removing these noise.
\end{abstract}

KeyWords: deblurring, image degradation, MSE,PSNR,

\section{Introduction}

Image restoration is the process that attempts to recover the image from its corrupted version. Restoration techniques are oriented toward modeling the degradation and applying the inverse process in order to recover the original image. Basically contrast stretching is considered an enhancement technique because it is based primarily on the pleasing accepts it might present to the viewer, whereas removal of image blur by applying a deblurring function is considered a restoration technique. The challenge to scientists, engineers and business people is to quickly extract valuable information from raw image data. This is the primary objective of image processing i.e. converting images to information. The following example introduces some basic image processing concepts. The example starts by reading an image into the MATLAB workspace. The example then performs some contrast adjustment on the image. Contrast adjustment includes the deblurring process and then adding a noises to that loaded image so that we can compare these different images after the filtration process. Image restoration is concerned with the reconstruction or estimation of blur parameters of the uncorrupted image from a blurred and noisy one.

\subsection{Image Degradation}

In degradation process, a degradation function $H$ that, together with an additive noise term $\eta(x, y)$, operates on an input image $f(x, y)$,to produce a degraded image $g(x, y)$. The objective of a restoration is to obtain an estimate $\hat{f}(x, y)$ of the original image.

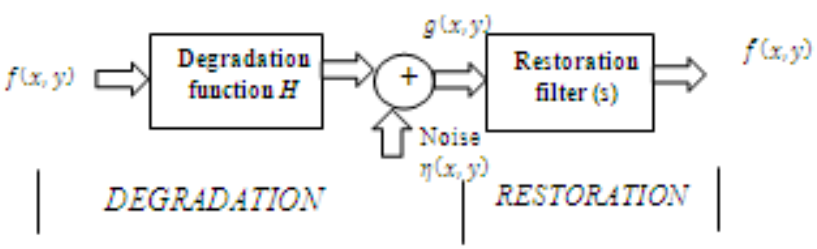

Fig -1:A model of the image degradation/restoration process.

Where, $\mathrm{H}$ is a linear, position -invariant process, then the degraded image is given in the spatial domain by

$\mathrm{g}(\mathrm{x}, \mathrm{y})=\mathrm{h}(\mathrm{x}, \mathrm{y}) * \mathrm{f}(\mathrm{x}, \mathrm{y})+\eta(\mathrm{x}, \mathrm{y}) \quad$ equation (1)

Where $h(x, y)$ is the spatial representation of the degradation function and, the symbol '*' indicated convolution. As we know that convolution is the spatial domain is analogous to multiplication in the frequency domain, so we may write the above equation in an equivalent frequency domain representation:

$\mathrm{G}(\mathrm{u}, \mathrm{v})=\mathrm{H}(\mathrm{u}, \mathrm{v}) \mathrm{F}(\mathrm{u}, \mathrm{v})+\mathrm{N}(\mathrm{u}, \mathrm{v})$ equation (2) 
Where the terms in capital letters are the Fourier transforms of the corresponding terms of the first equation. These two equation are the bases for most of the restoration of images.

Peak Signal to Noise Ratio (PSNR): One of the common reliable methods to measure the accuracy in the image processing field is the (PSNR), the peak signal to noise ratio for a grayscale image can compute using the following equation.

PSNR $=20 * \log _{10}\left(\frac{255}{\sqrt{\mathrm{MSE}}}\right)$ equation (3)

Mean square error (MSE): Where MSE can be calculated using the following equation:

MSE $=\frac{1}{\mathrm{M} * \mathrm{~N}} \sum_{\mathrm{x}=1}^{\mathrm{M}} \sum_{\mathrm{y}=1}^{\mathrm{N}} \mathrm{f}(\mathrm{x}, \mathrm{y})-\grave{\mathrm{f}}(\mathrm{x}, \mathrm{y})$ equation (4)

Where, $(M, N)$ are the dimensions of the image, $f(x, y)$ is the original image, $f^{\prime}(x, y)$ is the restoredimage. The higher PSNR value means the image has a better quality in the deblurred image. This metric helps to deliver an unbiased standard to compare diverse techniques.

Different Deblurring Process we used are: Motion Process, Disk Process, Unsharp Process, Sobel process, Log Process, Gaussian Process. And the filters we used are wiener filter, Median filter, and Ordinary filter. For comparing the two different images, we added different noises into the image. They are known as: salt $\&$ pepper noise, speckle noise, poison noise, gaussian noise

The following list shows the description for eachfilter type:

- Motion process - This filter type returns a filter to approximate, the linear motion of a camera by length pixels, with an angle of theta degrees in a counterclockwise direction.

- Disk process - Returns a circular averaging filter within the square matrix.

- Unsharp process - Returnsa unsharp contrast enhancement filter.

- Sobel process - Returnsa emphasizes horizontal edges using the smoothing effect by approximating a vertical gradient.

- Log process -This filter is Laplacian of Gaussian filter.

- Gaussian process - Returns a rotationally symmetric Gaussian lowpass filter.

\section{Proposed Work}

Image restoration techniques such as inverse filtering and Wiener Filtering can be considered as simple. Deblurring images with a known blur function is commonly done using the Wiener filter.

The proposed efforts have been utilized to compare the different deblurring techniques are shown using algorithm. This technique will capitalize on the statistics of the blurry image and the refined image estimate, in an iterative approach to converge on the correct seeing parameter.]direct restoration techniques The problem with such methods is that they require knowledge of the blur function that is point-spread function (PSF), which is, unfortunately, usually not available when dealing with image blurring.

\subsection{Results and Discussion:}

\subsubsection{Methodology:}

The following flow chart (methodology) describes the process of implementation of deblurring process and filtration. After this process we get our desired output and comparing the PSNR and MSE values of the outputs.

When an image is read in MATLAB workspace, the different deblurring methods are being used one by one. Let we firstly used motion process is implemented in original image and then after this process one noise is added into this deblurred image. After this, we firstly filter the deblurred image with any of the mention filters and we having its MSE and PSNR values, secondly we filtered the noisy image with same filter and similarly we having an its MSE and PSNR values. From all these values we compare the two different image result and which image is better. By comparing the different values of MSE and PSNR, by using the different filters, it is seen that, when we using the Gaussian deblurring Process with wiener filter the results are far better from other filters. 


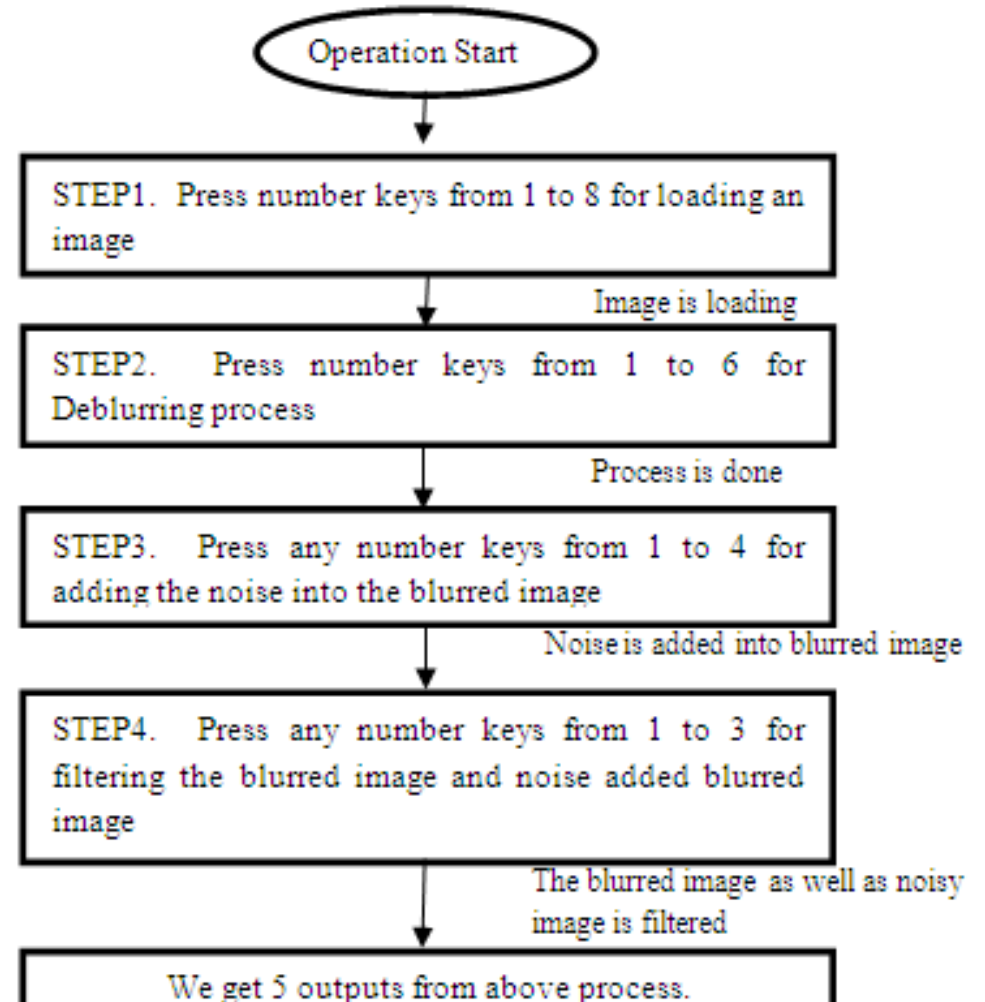

Fig -2: Methodology of the Project

For better result we need lower MSE and higher PSNR values. Now we comparing the two images by using the Gaussian deblurring process with gaussian noise in different filters.

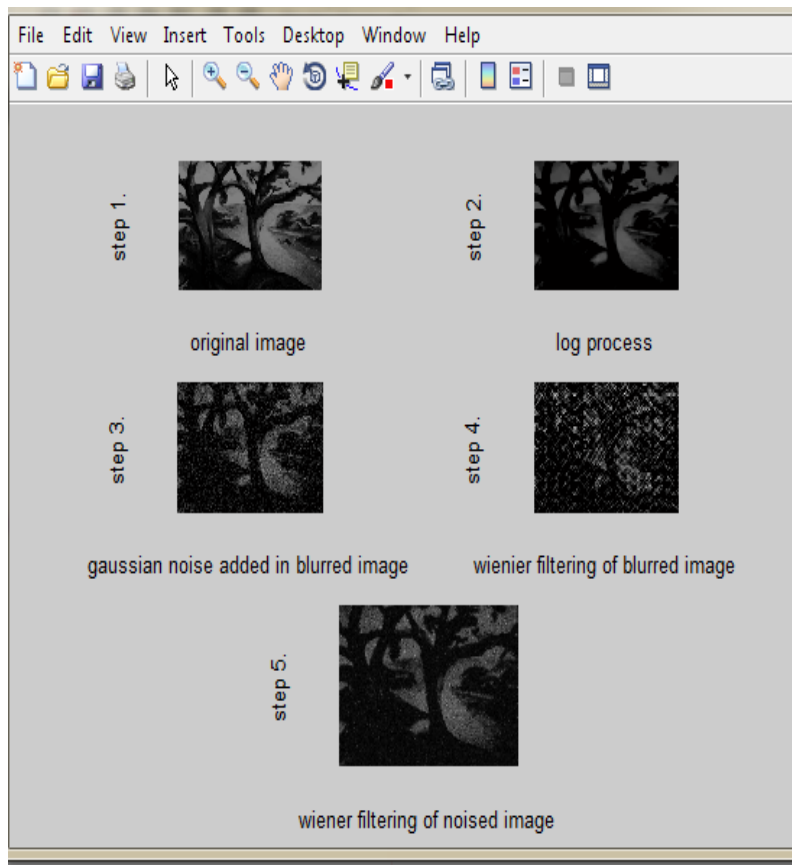

Fig -3: Log deblurring process with gaussian noise in Wiener filtering.

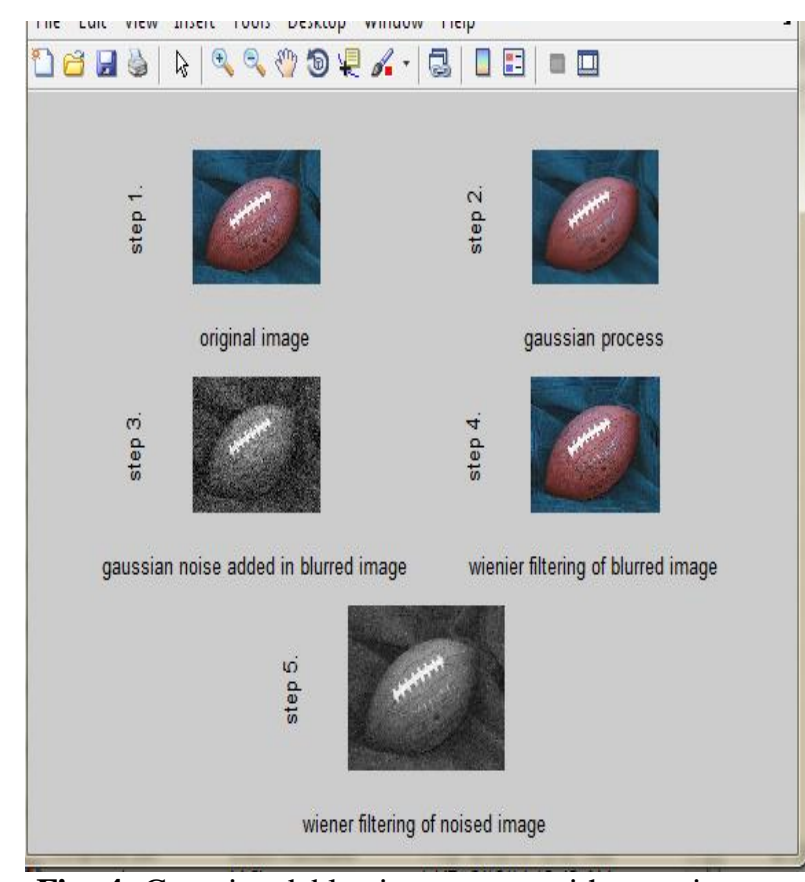

Fig -4: Gaussian deblurring process with gaussian noise in Wiener filtering. 


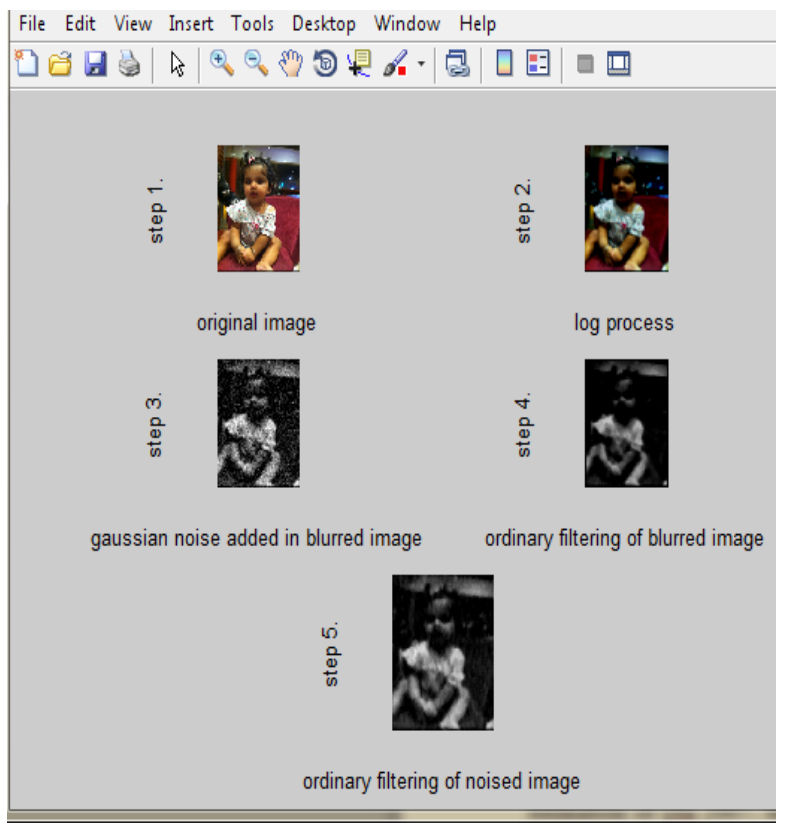

Fig -5: Log deblurring process with gaussian noise in Ordinary filtering.

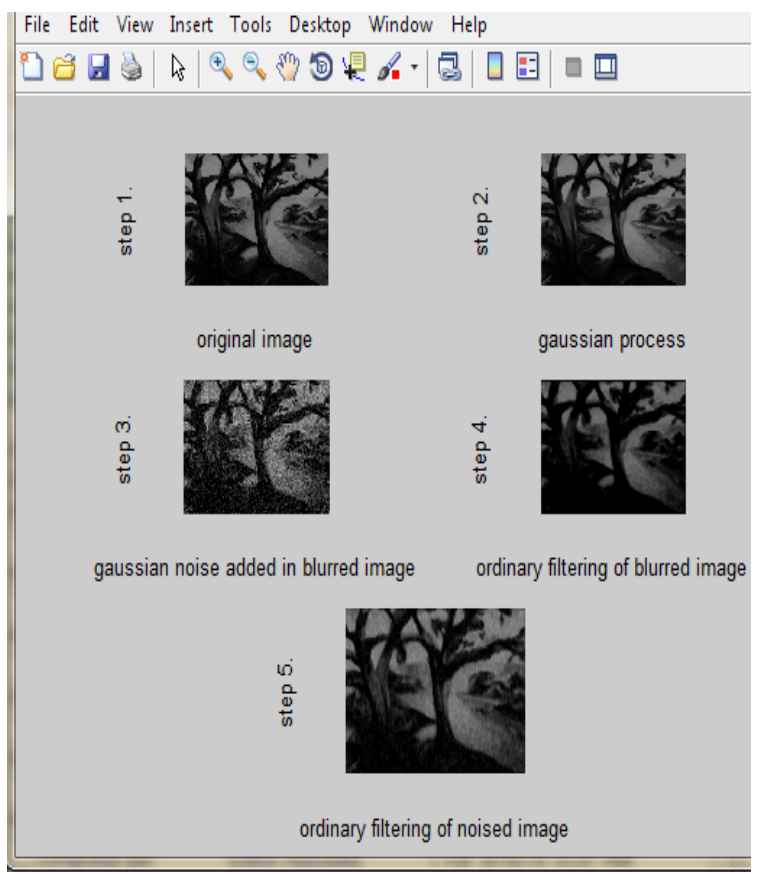

Fig -6: Gaussian deblurring process with gaussian noise in Ordinary filtering.

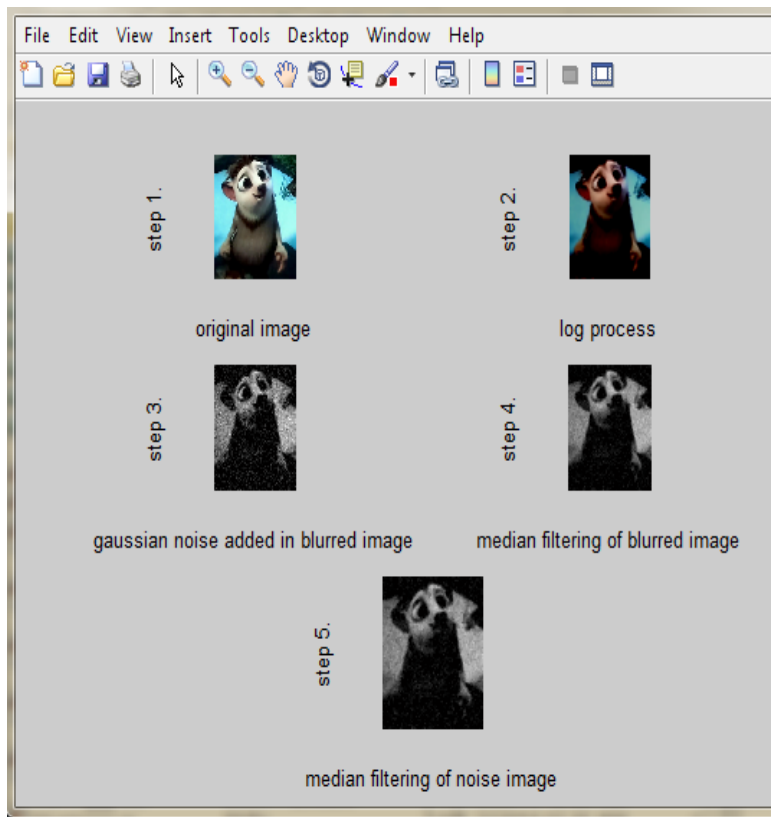

Fig -7: Log deblurring process with gaussian noise in Median filtering.

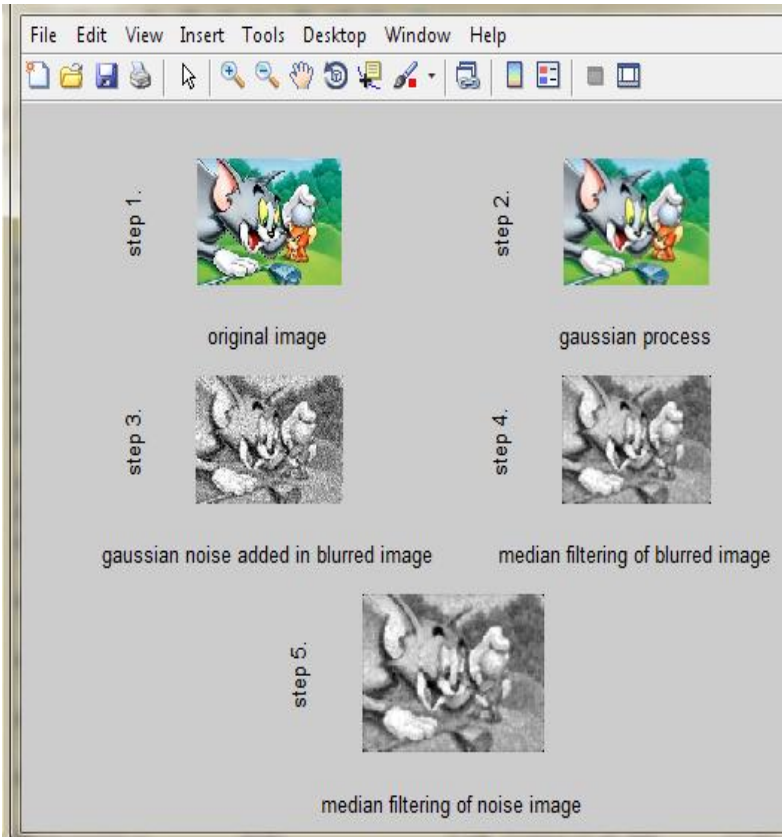

Fig -8: Gaussian deblurring process with gaussian noise in Median filtering.

The following tables shows the different PSNR and MSE values through which we can judge that which filter is best and comparing them.

Table 1 - PSNR in dB in Wiener Filtering

\begin{tabular}{|c|c|c|c|c|}
\hline Deblurring Methods Noise & Speckle noise & Salt \& Pepper noise & Poisson noise & Gaussian noise \\
\hline Motion Process & 59.5448 & 56.6984 & 54.7804 & 50.4465 \\
\hline Disk Process & 55.3720 & 54.4907 & 53.6547 & 55.5710 \\
\hline Unsharp Process & 52.9114 & 54.2014 & 53.8087 & 53.3154 \\
\hline Gaussian Process & 62.4521 & 49.943 & 63.0100 & 59.4788 \\
\hline
\end{tabular}


Table 2-PSNR in dB in Ordinary Filtering

\begin{tabular}{|c|c|c|c|c|}
\hline \begin{tabular}{|l|l} 
Deblurring Methods & Noise
\end{tabular} & Speckle noise & Salt \& Pepper noise & Poisson noise & Gaussian noise \\
\hline Motion Process & 38.4364 & 40.84887 & 41.9184 & 40.9500 \\
\hline Disk Process & 40.7439 & 45.7442 & 45.5759 & 40.4713 \\
\hline Unsharp Process & 37.6836 & 38.4473 & 40.3584 & 38.5415 \\
\hline Sobel Process & 37.0322 & 34.3100 & 37.1451 & 34.6314 \\
\hline Log Process & 41.7645 & 38.4883 & 45.3884 & 38.5371 \\
\hline Gaussian Process & 37.2425 & 43.6027 & 41.2532 & 38.9401 \\
\hline
\end{tabular}

Table 3 -PSNR in $\mathrm{dB}$ in Median Filtering

\begin{tabular}{|c|c|c|c|c|}
\hline \begin{tabular}{|l|l} 
Deblurring Methods Noise \\
\end{tabular} & Speckle noise & Salt \& Pepper noise & Poisson noise & Gaussian noise \\
\hline Motion Process & 49.7371 & 42.0637 & 50.8290 & 51.0116 \\
\hline Disk Process & 50.9572 & 40.1936 & 52.1178 & 50.0039 \\
\hline Unsharp Process & 43.0350 & 42.4453 & 43.4982 & 46.0546 \\
\hline Sobel Process & 37.7114 & 37.4848 & 41.7345 & 39.0547 \\
\hline Log Process & 50.1520 & 38.1229 & 48.384 & 41.1875 \\
\hline Gaussian Process & 47.1520 & 46.0855 & 50.8828 & 59.8618 \\
\hline
\end{tabular}

Table 4-MSE in wiener filtering

\begin{tabular}{|c|c|c|c|c|c|}
\hline Deblurring Methods & Noise & Speckle noise & Salt \& Pepper noise & Poisson noise & Gaussian noise \\
\hline Motion Process & & 0.0562 & 0.1083 & 0.1884 & 0.4538 \\
\hline Disk Process & & 0.1469 & 0.1800 & 0.2182 & 0.1404 \\
\hline Unsharp Process & & 0.2590 & 0.1924 & 0.2106 & 0.2360 \\
\hline Sobel Process & & -0.4103 & 0.3704 & -0.2243 & -0.0248 \\
\hline Log Process & & -0.1368 & 0.2922 & -0.1528 & -0.1797 \\
\hline Gaussian Process & & 0.0288 & 0.5125 & -0.0499 & 0.0571 \\
\hline
\end{tabular}

Table 5-MSE in ordinary filter

\begin{tabular}{|c|c|c|c|c|c|}
\hline Deblurring Methods & Noise & Speckle noise & Salt \& Pepper noise & Poisson noise & Gaussian noise \\
\hline Motion Process & & 7.2565 & 4.1638 & 3.2548 & 4.0678 \\
\hline Disk Process & & 4.2656 & 1.3488 & 1.4021 & 4.5418 \\
\hline Unsharp Process & & 8.6298 & 7.2383 & 4.6615 & 7.0831 \\
\hline Sobel Process & & 10.0265 & 18.7658 & 9.7692 & 19.4272 \\
\hline Log Process & & 3.3722 & 7.1703 & 1.1639 & 7.0902 \\
\hline Gaussian Process & & 9.5524 & 2.2085 & 3.7935 & 6.4619 \\
\hline
\end{tabular}

Table6 -MSE in Median filtering

\begin{tabular}{|c|c|c|c|c|c|}
\hline Deblurring Methods & Noise & Speckle noise & Salt \& Pepper noise & Poisson noise & Gaussian noise \\
\hline Motion Process & & 0.5378 & 3.1477 & 0.4183 & 0.4011 \\
\hline Disk Process & & 0.4061 & 4.8417 & 0.3109 & 0.5058 \\
\hline Unsharp Process & & 2.5169 & 2.8829 & 2.2623 & 1.2558 \\
\hline Sobel Process & & 8.5748 & 9.0341 & 3.3956 & 6.2935 \\
\hline Log Process & & 0.4888 & 7.7997 & 0.7349 & 3.8514 \\
\hline Gaussian Process & & 1.0028 & 1.2468 & 0.4131 & 0.0523 \\
\hline
\end{tabular}

Hence from the above comparisons, it is clear that wiener filter is the best. It shows better results from all other filters. It has high PSNR value and lower MSE.

\section{Conclusions}

This paper shows various approaches for image restoration based on deblurring methods. The performance of different deblurring methods and their experimental results shows that Gaussian process yield best result. The relative performance of various deblurring process with noise as well as filtering is carried out with an image by using MATLAB software. Also, the different filtering techniques are applied in this project which tells that wiener filtering gives better performance on the basis of PSNR, than median filter and ordinary filter.

\section{References}

[1] Sonia Saini, and LalitHimral, "Image processing using blind deconvolution deblurring Technique" , International Journal of Applied Engineering and Technology ISSN: 2277-212X (Online) An Open Access, Online International Journal Available at http://www.cibtech.org/jet.htm 2014 Vol. 4 (2) April-June, pp.115-124/S

[2] Mr. RohitVerma, Dr. Jahid Ali, "A Comparative Study of Various Types of Image Noise and Efficient Noise Remov-al Techniques" International Journal of Advanced Research in Computer Science and Software Engineering 3(10), October - 2013.

[3] Image Restoration Technique with Non Linear Filter CharuKhare and Kapil Kumar Nagwanshi International Journal of Advanced Science and Technology Vol. 39, February, 2012.

[4] Zohair Al-Ameen, GhazaliSulong, and Md. Gapar Md. Johar, "International Journal of Advanced Science and Technology",Vol. 44, July, 2012, A Comprehensive Study on Fast image Deblurring Techniques. 
[5] D. Krishnan, Member, IEEE, Ping Lin, and Andy M. Yip, “A Primal-Dual Active-Set Method for Non-Negativity Constrained Total Variation Deblurring Problems", IEEE transactions on image processing, vol. 16, no. 11, november 2007.

[6] Yuan, L., Sun, J., quan, L., and Shum, H.-Y. 2007. "Image Deblurring with Blurred/Noisy Image Pairs" in SIGGRAPH

[7] Alex Rav-Acha, ShmuelPeleg, "Two motion-blurred images are better than one", Pattern Recognition Letters 26 (2005) 311-317.

[8] Gonzalo R. Arce,Petros Maragos ,YrjoNeuvo ,Ioannis Pitas , "Guest Editorial Introduction to the Special Issue on Nonlinear Image Processing", IEEE transactions on image processing, vol. 5, no. 6, june 1996.

[9] Lucy, L. 1974, "Bayesian-based iterative method of image restoration", Journal of Astronomy 79, 745-754.

[10] Qi Shan, JiayaJia, AseemAgarwala, "High-quality Motion Deblurring from a Single Image", To appear in the ACM SIGGRAPH conference proceedings.

[11] M. Filipovi'c, A. Juki'c, "Restoration of Images Corrupted by Mixed Gaussian-Impulse Noise by Iterative soft-hard thresholding".

[12] Rob Fergus, Barun Singh, Aaron Hertzmann, Sam T. Roweis, William T. Freeman, "Removing Camera Shake from a Single Photograph".

[13] http://www.mathworks.com. 